\title{
$\begin{array}{ll}\text { Research Square } & \begin{array}{l}\text { Preprints are preliminary reports that have not undergone peer review. } \\ \text { They should not be considered conclusive, used to inform clinical practice, } \\ \text { or referenced by the media as validated information. }\end{array}\end{array}$
}

\section{Development and Validation of Radiomics Signatures to Predict KRAS Mutation Status Based on Triphasic Enhanced Computed Tomography in Patients with Colorectal Cancer}

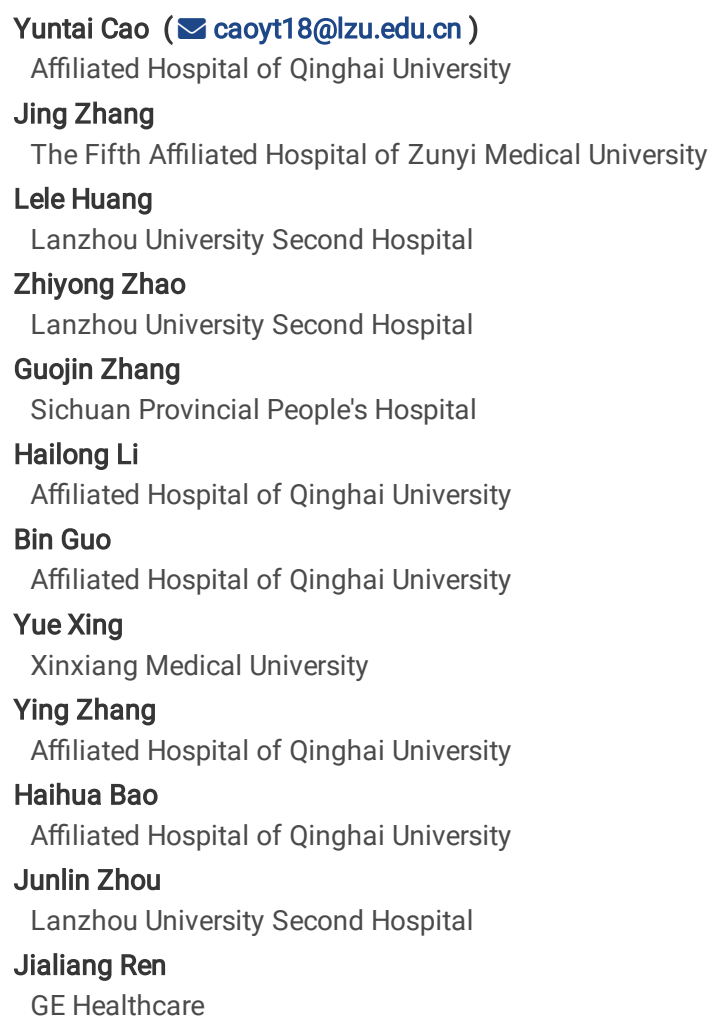




\section{Abstract}

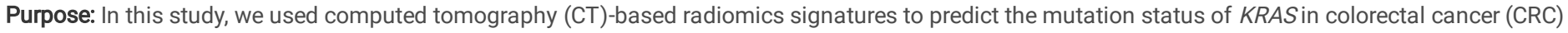
patients.

Methods: This study involved 447 patients who underwent KRAS mutation testing and preoperative triphasic enhanced CT. They were categorised into training $(n=313)$ and validation cohorts $(n=134)$ in the ratio 7:3. Radiomics features were extracted from CT imaging. The Boruta algorithm was used to retain the features closely associated with KRAS mutation. Multivariate logistic regression was used to develop radiomics, clinical, and combined clinical-radiomics models for KRAS mutation. The receiver operating characteristic curves were used to evaluate the predictive performance and clinical usefulness of each model.

Results: Fourteen radiomics features were retained as the as final signatures for predicting KRAS mutations. Delayed phase models showed superior predictive performance compared to arterial phases models or venous phase models. The clinical-radiomics fusion model showed excellent performance, with an AUC, sensitivity and specificity were $0.772,0.792$ and 0.646 in the training cohort, while $0.755,0.724$ and 0.684 in the validation cohort, respectively.

Conclusions: The clinical-radiomics fusion model can be used as a potential imaging marker for preoperative detection of $K R A S$ mutation status.

\section{Introduction}

Colorectal cancer (CRC) is the second leading cause of cancer-related deaths worldwide and causes almost 881,000 deaths every year ${ }^{1}$. The incidence of colorectal cancer is approximately 3-fold higher in developed countries than in developing countries. However, as the developing countries become richer, increasing trends is likely to be seen in these countries ${ }^{2}$. Kirsten rat sarcoma (KRAS) viral oncogene homologue is a $\mathrm{G}$ protein, which occurs in $40-50 \%$ cases of CRCs. Following the mutation in KRAS gene, the mutant protein activates downstream mitogen-activated protein kinase (MAPK) pathway, subsequently leading to uncontrolled cell proliferation and malignancy ${ }^{3}$. National Comprehensive Cancer Network (NCCN) Clinical Practice Guidelines have explicitly indicated that CRC patients with KRAS mutations are resistant to anti-EGFR monoclonal antibody therapy ${ }^{4}$. Therefore, KRAS mutation testing is crucial for individualised and effective treatment of CRC.

Generally, pathologic specimens obtained via invasive procedures such as colonoscopy or surgery are usually required for the identification of KRAS mutation status. However, the presence of extensive heterogeneity in CRC archival samples represents a major limitation of the histological approach ${ }^{5}$. Additionally, postoperative tissue specimens might not be obtained for testing from patients with metastatic CRC specimens ${ }^{6}$. Furthermore, biopsy testing might not be an effective approach to determine the mutational status of KRAS due to poor DNA quality ${ }^{7}$. Therefore, it is necessary to develop a non-invasive and easy-to-use method to identify KRAS mutation status.

Several studies have demonstrated the use of medical imaging technology, such as fluorine-18 fludexyglucose (18F-FDG) positron emission tomography (PET) and magnetic resonance imaging, in the prediction of $K R A S$ status ${ }^{8,9}$. However, these studies involved small sample sizes and lacked validation. Radiomics provides a variety of parameters for quantitative analysis, and these parameters have been widely used in cancer diagnosis, classification, and prediction ${ }^{10}$. A previous study demonstrated a significant correlation between a CT-based radiomics signature and KRAS/NRAS/BRAF mutations in CRC patients ${ }^{11}$. However, this study involved a small sample size and was only performed in the venous phase. Moreover, the superiority of venous phase as compared to arterial or delay phases in the prediction of KRAS mutation status in CRC patients remains to be confirmed. The aim of this study was to investigate whether a CT-based radiomics signature could identify KRAS mutation status in CRC patients and whether the venous phase is superior to arterial and delay phases in the prediction of KRAS mutation status in CRC patients.

\section{Results}

\section{Clinical Characteristics}

This study involved a total of $447 \mathrm{CRC}$ patients in the final analysis, including 263 men (58.8\%) and 184 women (41.2\%), with an average age of $58.93 \pm 12.85$ years. Among the 447 patients, 207 patients contained mutated KRAS and 240 patients contained wild-type KRAS. We used stratified sampling to categorise the study cohort into a training cohort $(n=313)$ and a validation cohort $(n=134)$ in the ratio of $7: 3$. The training cohort and validation cohort were used for model building and internal validation, respectively. Patient and tumor characteristics in the training cohort are listed in Table 1. 
Table 1

Demographic and Clinical Characteristics of and CT Findings for CRC (mean \pm SD or no. (\%).

\begin{tabular}{|c|c|c|c|}
\hline \multirow[t]{2}{*}{ Characteristics $^{1}$} & \multicolumn{3}{|c|}{ Training cohort $(n=313)$} \\
\hline & KRAS WT $(n=164)$ & KRAS MT (n=149) & P value $^{2}$ \\
\hline Age (years) & $57.58 \pm 12.81$ & $60.50 \pm 12.75$ & 0.023 \\
\hline Gender Female & $63(38.4 \%)$ & $66(44.3 \%)$ & 0.291 \\
\hline Male & $101(61.6 \%)$ & $83(55.7 \%)$ & \\
\hline Tumor Location Left & $115(70.1 \%)$ & $100(67.1 \%)$ & 0.567 \\
\hline Right & 49 (29.9\%) & 49 (32.9\%) & \\
\hline CEA level Normal & $101(61.6 \%)$ & $72(48.3 \%)$ & 0.018 \\
\hline Abnormal & $63(38.4 \%)$ & 77 (51.7\%) & \\
\hline CA125 level Normal & $150(91.5 \%)$ & $135(90.6 \%)$ & 0.790 \\
\hline Abnormal & $14(8.5 \%)$ & $14(9.4 \%)$ & \\
\hline CA199 level Normal & $120(73.2 \%)$ & $90(60.4 \%)$ & 0.016 \\
\hline Abnormal & $44(26.8 \%)$ & 59 (39.6\%) & \\
\hline cT stage T1 & $2(1.2 \%)$ & $9(6.0 \%)$ & 0.023 \\
\hline T2 & $21(12.8 \%)$ & 29 (19.5\%) & \\
\hline T3 & $107(65.2 \%)$ & $90(60.4 \%)$ & \\
\hline T4 & $34(20.7 \%)$ & $21(14.1 \%)$ & \\
\hline cN stage NO & $100(61.0 \%)$ & $81(54.4 \%)$ & 0.281 \\
\hline N1 & $37(22.6 \%)$ & $33(22.1 \%)$ & \\
\hline N2 & 27 (16.5\%) & 35 (23.5\%) & \\
\hline Maximum diameter (mm) & $22.42 \pm 9.95$ & $21.85 \pm 8.88$ & 0.825 \\
\hline \multicolumn{4}{|c|}{${ }^{1}$ Continuous variables were expressed as mean \pm standard deviation; Classification variables were represented by no. (\%); } \\
\hline \multicolumn{4}{|c|}{$\begin{array}{l}2 \text { Student's t-test or Mann-Whitney U test was used to compare continuous variables; Chi-squared test or Fisher's exact test was used to compare } \\
\text { categorical variables. }\end{array}$} \\
\hline
\end{tabular}

\section{Predictive Performance of the Clinical Model}

In the training cohort, the clinical characteristics age, CEA, CA199, and CT stage were found to be significantly different statistically $(P<0.05)$, and the other characteristics not significantly different $(P>0.05)$ between mutated KRAS and wild-type KRAS groups (Table 1). Therefore, after multivariate analyses, clinical characteristics including age, CEA and cT stage were selected as independent predictors of KRAS mutation and enrolled into clinical model. The clinical model showed lower performance in predicting KRAS mutation both in the training cohort and the validation cohort, with the AUC being 0.654 ( $95 \% \mathrm{Cl}$, $0.593-0.714)$ in the training cohort and $0.575(95 \% \mathrm{Cl}, 0.478-0.672)$ in the validation cohort (Table 2). The accuracy, sensitivity, and specificity were 0.617 , 0.664 , and 0.573 (training cohort) and $0.552,0.552$, and 0.553 (validation cohort), respectively.

Table 2. Predictive performance of different models in training and validation cohorts. 


\begin{tabular}{|c|c|c|c|c|c|c|c|c|c|}
\hline \multirow[t]{2}{*}{ Feature_num } & \multirow[t]{2}{*}{ Methods } & \multicolumn{6}{|c|}{ Training cohort } & \multicolumn{2}{|c|}{ Validation cohort } \\
\hline & & AUC & Accuracy & Sensitivity & Specificity & PPV & NPV & AUC & Accuracy \\
\hline 4 & A & $\begin{array}{l}0.711(0.654- \\
0.767)\end{array}$ & $\begin{array}{l}0.649(0.593- \\
0.701)\end{array}$ & $\begin{array}{l}0.758(0.670- \\
0.837)\end{array}$ & $\begin{array}{l}0.549(0.446- \\
0.662)\end{array}$ & $\begin{array}{l}0.604(0.574- \\
0.628)\end{array}$ & $\begin{array}{l}0.714(0.670- \\
0.751)\end{array}$ & $\begin{array}{l}0.723(0.637- \\
0.809)\end{array}$ & $\begin{array}{l}0.679(0.593 \\
0.757)\end{array}$ \\
\hline 3 & V & $\begin{array}{l}0.692(0.634- \\
0.750)\end{array}$ & $\begin{array}{l}0.639(0.583- \\
0.692)\end{array}$ & $\begin{array}{l}0.597(0.446- \\
0.694)\end{array}$ & $\begin{array}{l}0.677(0.557- \\
0.751)\end{array}$ & $\begin{array}{l}0.627(0.556- \\
0.661)\end{array}$ & $\begin{array}{l}0.649(0.604- \\
0.673)\end{array}$ & $\begin{array}{l}0.673(0.582- \\
0.764)\end{array}$ & $\begin{array}{l}0.657(0.57 C \\
0.737)\end{array}$ \\
\hline 7 & $\mathrm{D}$ & $\begin{array}{l}0.752(0.699- \\
0.806)\end{array}$ & $\begin{array}{l}0.709(0.656- \\
0.759)\end{array}$ & $\begin{array}{l}0.638(0.473- \\
0.731)\end{array}$ & $\begin{array}{l}0.774(0.612- \\
0.837)\end{array}$ & $\begin{array}{l}0.720(0.656- \\
0.746)\end{array}$ & $\begin{array}{l}0.702(0.650- \\
0.718)\end{array}$ & $\begin{array}{l}0.746(0.662- \\
0.830)\end{array}$ & $\begin{array}{l}0.687(0.601 \\
0.764)\end{array}$ \\
\hline 11 & Radiomics & $\begin{array}{l}0.754(0.701- \\
0.807)\end{array}$ & $\begin{array}{l}0.700(0.646- \\
0.750)\end{array}$ & $\begin{array}{l}0.738(0.607- \\
0.805)\end{array}$ & $\begin{array}{l}0.665(0.530- \\
0.748)\end{array}$ & $\begin{array}{l}0.667(0.622- \\
0.686)\end{array}$ & $\begin{array}{l}0.736(0.690- \\
0.759)\end{array}$ & $\begin{array}{l}0.775(0.697- \\
0.854)\end{array}$ & $\begin{array}{l}0.701(0.61 \epsilon \\
0.777)\end{array}$ \\
\hline 3 & Clinical & $\begin{array}{l}0.654(0.593- \\
0.714)\end{array}$ & $\begin{array}{l}0.617(0.560- \\
0.671)\end{array}$ & $\begin{array}{l}0.664(0.516- \\
0.744)\end{array}$ & $\begin{array}{l}0.573(0.420- \\
0.655)\end{array}$ & $\begin{array}{l}0.586(0.523- \\
0.613)\end{array}$ & $\begin{array}{l}0.653(0.579- \\
0.682)\end{array}$ & $\begin{array}{l}0.575(0.478- \\
0.672)\end{array}$ & $\begin{array}{l}0.552(0.464 \\
0.638)\end{array}$ \\
\hline 4 & $\begin{array}{l}\text { Clinical- } \\
\text { radiomics }\end{array}$ & $\begin{array}{l}0.772(0.720- \\
0.823)\end{array}$ & $\begin{array}{l}0.716(0.662- \\
0.765)\end{array}$ & $\begin{array}{l}0.792(0.673- \\
0.866)\end{array}$ & $\begin{array}{l}0.646(0.514- \\
0.720)\end{array}$ & $\begin{array}{l}0.670(0.634- \\
0.690)\end{array}$ & $\begin{array}{l}0.774(0.731- \\
0.792)\end{array}$ & $\begin{array}{l}0.755(0.674- \\
0.836)\end{array}$ & $\begin{array}{l}0.701(0.61 \epsilon \\
0.777)\end{array}$ \\
\hline
\end{tabular}

Radiomics, fusion of radiomics features of arterial phase, venous phase, and delayed phase; Clinical, fusion of clinical and imaging characteristics; Clinicalradiomics, fusion of clinicalradiological features and radiomics features; AP, radiomics model of arterial phase; DP, radiomics model of delayed phase; VP, radiomics model of venous phase. Abbreviations: AUC: area under the curve; NPV: negative predictive value; PPV: positive predictive value.

\section{Radiomics signature building and discrimination performance assessment}

Finally, 4, 3 and 7 radiomics features were selected as the final signatures. The feature names and distributions are listed in Table 3 . Following stepwise regression analysis, three features were removed after combining the AP, VP and DP radiomics features. Four models were built based on the above radiomics signatures for preoperatively predicting KRAS mutation. The AUC, accuracy, sensitivity, specificity, PPV, and NPV are listed in Table 2. The DP model had the optimal predictive performance than AP model or VP model both in training and validation cohorts (Figure 3A-B, Table 2). In the training cohort, the predictive AUC of KRAS mutations in AP, VP and DP models were $0.711,0.692$ and 0.752 , respectively. In the validation cohort, the AUC of the three models were 0.723 , 0.673 and 0.746 , respectively. The Radiomics model combined by triphasic enhanced CT phases showed moderate KRAS mutation prediction performance, with AUC, accuracy, sensitivity, specificity, PPV and NPV of $0.754,0.700,0.738,0.665,0.667$ and 0.736 in the training cohort, respectively, while AUC, accuracy, sensitivity, specificity, PPV and NPV in the validation cohort were $0.775,0.701,0.707,0.697,0.641$ and 0.757 , respectively (Figure 3A-B, Table 2).

Table 3

The final signatures selected from 3D radiomics features.

\begin{tabular}{lll} 
Arterial phase & Venous phase & Delayed phase \\
(4) & $(3)$ & $(7)$ \\
\hline A_original_shape_Elongation & V_original_shape_Maximum2DDiameterSlice & D_original_shape_Elongation \\
\hline A_wavelet.HLL_firstorder_Skewness & V_original_shape_Sphericity & D_original_shape_Sphericity \\
\hline A_wavelet.HHH_glszm_GrayLevelNonUniformityNormalized & V_wavelet.HLL_firstorder_Median & D_wavelet.HLL_glcm_Idn \\
\hline A_wavelet.LLL_glcm_MCC & & D_wavelet.LLL_glcm_Idn \\
& & D_original_shape_Maximum3DDiameter \\
& & D_original_shape_SurfaceArea \\
& D_wavelet.HLL_gldm_SmallDendenceLowGrc
\end{tabular}

\section{Predictive Performance of the Combined Model}

As shown in Table 2 and Figure 3, we developed a clinical-radiomics model incorporating three clinical factors (age, CEA and cT stage) and seven DP radiomics signatures. The clinical-radiomics model showed excellent predictive ability for KRAS mutations. The clinical-radiomics fusion model showed superior predictive performance for KRAS mutations than either the clinical model or the radiomics model alone, the AUC values of the clinical-radiomics model were $0.772(95 \% \mathrm{Cl}, 0.720-0.823)$ in the training cohort and $0.755(95 \% \mathrm{Cl}, 0.674-0.836)$ in the validation cohort. The calibration curve of each model showed favourable agreement between prediction and observation in predicting the risk of KRAS mutation (Figure 4A-B). 
The DCA curves for the clinical model, radiomics models, and clinical-radiomics model is presented in Figure 5A-B. The combined clinical-radiomics model achieved more clinical utility in predicting the KRAS mutation than the clinical model and other radiomics models. The DCA curves of the clinical-radiomics model demonstrated that when the threshold probability of a patient or doctor ranged between $20 \%$ and $65 \%$, the use of the clinical-radiomics nomogram adds greater benefit for KRAS mutation prediction than the treat-all-patients scheme or the treat-none scheme in training and validation cohorts.

\section{Discussion}

In this study, clinical, radiomics, and clinical-radiomics model were developed for the preoperative prediction of KRAS mutations. We answered a question that the DP model had a higher outstanding performance than the AP or VP models. Additionally, the clinical-radiomics model showed higher predictive performance than the clinical model or radiomics model alone. The calibration curve and decision curve of clinical-radiomics model showed excellent model stability and actual benefit.

KRAS mutations can lead to continuous activation of the EGF/RAS/RAF/ERK signalling pathway without the regulation of EGFR, gradually leading to increased cell proliferation and decreased apoptosis ${ }^{12-14}$. Colorectal cancer with KRAS mutation is a negative marker for anti-EGFR targeted drugs ${ }^{15}$. Numerous studies ${ }^{16,17}$ have used ${ }^{18} \mathrm{~F}$-FDG PET/CT to investigate the association between KRAS mutation and $18 \mathrm{~F}$-FDG uptake and demonstrated that cells with KRAS mutation had higher 18F-FDG uptake than those with wild-type KRAS. However, there was no correlation observed between them according to a study by Riklis et al. ${ }^{18}$. The major clinical use of PET/CT in CRC is to detect potentially curable metastases. Yang et al. ${ }^{11}$ proposed a CT-based radiomics model to identify KRAS/NRAS/BRAF mutation in CRC and found a relatively high predictive performance. However, this study defined the positive group based on mutations in any of KRAS/NARS/BRAF, which would complicate the clinical application.

During the image preprocessing stage, the LoG filter and wavelet filter were applied to process the original image. The LoG filter can smoothen the image and improve the efficiency of capturing phenotypic features related to tumor heterogeneity ${ }^{19}$. The wavelet filter could disassemble the frequency signal of the image to extract edges and substantial features of tumor more effectively. This study finally screened out 25 radiomics features as the radiomics signatures of the AP, VP, DP, and triphasic enhanced combined phase. The features with wavelet filtering accounted for $52 \%(13 / 25)$ of the total features. This shows that the wavelet filter is very important for extracting features related to KRAS mutations status, while the features with LoG filters are not used as radiomics signatures for predicting KRAS mutations, indicating that the features extracted from the LoG filter images were weakly correlated with $K R A S$ mutations.

Multivariable regression analysis combined with the radiomics signatures of triphasic enhanced phases, 11 radiomics features were retained as key features for identifying KRAS mutation status (Table 3), including 5 texture features: A_wavelet.HHH_glszm_GrayLevelNonUniformityNormalized, A_wavelet.LLL_glcm_MCC, D_wavelet.HLL_glcm_Idn, D_wavelet.HLL_gldm_SmallDendenceLowGrayLevelEmphasis, D_wavelet.LLL_glcm_Idn. Texture features are microscopic descriptions of tumors, reflecting the interaction between adjacent pixels, and reflect tumor heterogeneity ${ }^{20}$. These features are not easily identified by the human visual system and cannot be interpreted as having a clear meaning. Previous studies have shown that texture features may be associated with tumor microenvironment reflecting tumor heterogeneity, presence of hypoxia or angiogenesis ${ }^{21-23}$. A studies ${ }^{24}$ found that $K R A S$ mutations were associated with higher texture characteristic values (Gskewness and SDs), indicating that mutated KRAS had more tumor heterogeneity than wild-type KRAS. The radiomics score values of texture features (A_wavelet. LLL_glcm_MCC, D_wavelet. HLL_gldm_SmallDendenceLowGrayLevelEmphasis) in the KRAS mutation group was higher than that in the wild group, which suggests that tumor tissue is more tumor heterogeneous in the ROI range. Combined with the results of this study and other studies, it is shown that texture features can be used as noninvasive imaging markers for predicting KRAS mutations status.

In addition, five morphological features (A_original_shape_Elongation\V_original_shape_Maximum2DDiameterSlice[D_original_shape_Elongation[ D_original_shape_Maximum3DDiameterID_original_shape_Sphericity) were also closely correlated with KRAS mutations, suggesting that the morphological characteristics of mutant KRAS and wild-type KRAS tumor were significantly different, which was consistent with previous literature. A study ${ }^{19}$ found morphological features (Elongation and flatness) were closely associated with KRAS mutations in rectal cancer. A study ${ }^{9}$ explored the correlation between $K R A S$ mutations in rectal cancer and tumor morphology on magnetic resonance images, the study found that the average axial/longitudinal ratio of $K R A S$ mutations rectal cancer was greater than that of $K R A S$ wild-type tumor $(0.46 \pm 0.29$ vs. $0.36 \pm 0.20, P=0.009)$.

Among the triphasic enhanced phase models of KRAS mutation prediction in the training cohort, the DP model showed the highest performance, with an AUC value of 0.752 , followed by 0.711 in the AP model and 0.692 in VP model. To our knowledge, this is the first time that the triphasic enhanced CT radiomics has been used in KRAS mutation prediction. Although the VP is the most commonly used phase in gastrointestinal radiomics research, contrary to the results assumed in this study, the enhancement phase with the best predictive performance was the DP rather than the VP. One reason for the high predictive performence of the DP model is the possibility of high content and uniform distribution of contrast agent in the DP lesions ${ }^{25}$. Another reason for the high predictive performance of the DP model may be that the ROI range of tumors in the DP images is larger than that in the AP and VP25.

In terms of clinical characteristics, Age, CEA and CA199 were independent predictors for KRAS mutation. In this study, KRAS mutations patients were older than KRAS wild-type patients, and the difference was statistically significant $(\mathrm{P}<0.05)$, this result is consistent with the previous literature ${ }^{26}$. CEA and $C A 199$ were significantly higher for the mutated KRAS group than for the wild-type KRAS group in our study. Our finding is in line with those from previous studies $^{27,28}$. Both KRAS mutation and elevated serum levels of CEA and CA199 are associated with more aggressive biological behaviour in CRC patients ${ }^{29-31}$. A correlation between KRAS mutations and higher CEA and CA199 levels suggests that genetic alterations may have independent influences on CRC development, thus resulting in increased tumor biomarkers ${ }^{32}$.

Triphasic enhanced CT is often conducted in gastrointestinal tumors CT examination. The AP is used for tumor detection, the VP is used to differentiate the tumor from adjacent organs, and the DP is used to determine the depth of tumor invasion ${ }^{33}$. As for radiation dose, the average DLP value of triphasic enhanced scans was $1917.52 \pm 152.31 \mathrm{mGy} \mathrm{cm}$, which is slightly higher than the diagnostic reference level (DRL) for adults (1490 mGy cm) published by

Page 5/13 
China's National Health Industry standard (WS/T 637-2018) ${ }^{34}$. Application of new techniques such as multi-model iterative reconstruction technology could effectively reduce the radiation dose in clinical practise ${ }^{35}$.

Our study should be interpreted after considering several limitations. First, 269 patients were excluded because they did not meet the inclusion and exclusion criteria, which inevitably produced selection bias. Second, our study only included a single team with an internal validation cohort. The reproducibility should be addressed in future multi-centre studies. Third, due to the irregular shape of some tumors, the ROI delineation process is difficult and time-consuming. In future studies, it will be very necessary to develop an automated or semi-automated tool to achieve effective and automatic tumor segmentation. Finally, in this study, we used different imaging instruments and acquisition parameters to complete the CT scanning. The influence of different instruments and different parameters on radiomics features is obvious. Therefore, it is important to standardise scanning protocols in different instruments and different institutions.

\section{Conclusion}

In conclusion, Triphasic enhanced CT radiomics models was constructed to predict KRAS mutation status in colorectal cancer, and the results showed that the AP, VP and DP models could better predict KRAS mutation status in the training cohort and validation cohort. The DP models showed a higher predictive performance compared to AP or VP models. Additionally, the clinical-radiomics model that incorporates both clinical risk factors and radiomics features of DP images showed good performance in predicting KRAS mutations. The clinical-radiomics fusion model can be used as a potential imaging marker for preoperative detection of KRAS mutation status, and guide the selection of molecular targeted drug therapy for CRC.

\section{Methods \\ Patients}

\section{Ethical approval}

had obtained by the medical ethics committee of Lanzhou University Second Hospital for this retrospective study, and the informed consent requirement was waived. All methods were carried out in accordance with relevant guidelines and regulations. For the primary cohort of this study, we analysed the institutional database in our hospital between March 2014 and June 2020 to identify eligible patients with confirmed cases of CRC who underwent curative resection. A total of 447 patients met the inclusion criteria in our study. The inclusion criteria were set based on the following factors: (1) pathologically identified cases of primary CRC adenocarcinoma; (2) patients who underwent KRAS mutation status testing prior to the treatment; (3) pre-treatment abdominal triphasic enhanced CT with a reconstruction slice thickness of $1.25 \mathrm{~mm}$. The exclusion criteria were set based on the following factors: (1) abdominal triphasic enhanced CT was not performed before surgery or the interval between abdominal triphasic enhanced CT and surgery was > two weeks; (2) CRC patients who have received any anticancer treatment prior to the collection of pathological tissue samples; (3) insufficient CT quality for qualitative and quantitative analyses; (4) clinical information incomplete; (5) occurrence of intussusception in the area where the tumor was located. Figure 1 shows the flow diagram of the recruitment pathway. Patients were categorised into training cohort and validation cohort in 7:3 ratio.

\section{Clinical-pathologic characteristics and semantic features}

Baseline clinicopathological characteristics included age, sex, tumor location, KRAS mutation status, CEA level (threshold value $\geq 5 \mathrm{ng} / \mathrm{mL},<5 \mathrm{ng} / \mathrm{mL}$ ), CA125 level (threshold value $\geq 35 \mathrm{U} / \mathrm{ml},<35 \mathrm{U} / \mathrm{ml}$ ), and CA199 level (threshold value $\geq 37 \mathrm{U} / \mathrm{ml},<37 \mathrm{U} / \mathrm{mL}$ ). These data were collected from medical records, blindly and independently by two radiologists to ensure the accuracy of the extracted data. Two experienced gastrointestinal radiologists ( $\mathrm{Y} T \mathrm{C}$ and $\mathrm{J} Z$ ), analysed the images (including tumor location, maximum diameter, cT stage, and cN stage). Both radiologists were blinded to the patient's clinicopathological data. The maximum tumor thickness was defined as the maximum diameter perpendicular to the long axis of the cross-sectional image. Clinical tumor stage (cT stage) and clinical node stage (cN stage) were identified according to the 8th edition of the American Joint Committee on Cancer Staging system ${ }^{36}$.

\section{KRAS mutation evaluation}

Formalin-fixed tumor tissue samples were obtained following CRC operations and confirmed that the specimens used to extract DNA are clearly infiltrated by the tumor. DNA was extracted from formalin-fixed tumor sections. KRAS mutation status (exons 2, 3, and 4) was detected via polymerase chain reaction (PCR).

\section{CT Image Acquisition and segmentation}

Abdominal triphasic enhanced CT scans were performed on a Discovery CT 750 HD scanner (GE Healthcare, Waukesha, WI) and iCT 256 scanner (Philips, Amsterdam, Netherlands). The scanning parameters are listed in supplementary Table S1. Enhanced CT scanning was performed using a high-pressure dualcylinder syringe to inject intravenous iohexol $(1 \mathrm{~mL} / \mathrm{kg})$ through the median cubital vein with an injection rate of $3.5-4.5 \mathrm{ml} / \mathrm{second}$. Following the injection of contrast medium, arterial phase (AP), venous phase (VP), and delayed phase (DP) were scanned at 25-30 s, 60-70 s, and 120-150 s, respectively.

The original images of AP, VP and DP were stored in the corresponding folders in DICOM format. Two gastrointestinal radiologists (reader $1 \mathrm{Y}$ T C and reader 2 $\mathrm{J}$ Z) performed three-dimensional (3D) radiomics segmentation on AP, VP, and DP using ITK-SNAP software (version 3.6.0; www.itksnap.org). Reader 1 segmented 247 cases, reader 2 segmented the other 200 cases.

For 3D radiomics segmentation, the ROI was manually delineated on each slice of the tumor. Air and faeces in the intestinal tract, and pericolonic fat were carefully excluded from the contours (Figure 2). Finally, each patient generated three ROIs (AP ROI, VP ROI and DP ROI). To evaluate inter-observer 
reproducebility and robustness of feature extraction, reader 1 and reader 2 randomly select 30 patients and do the manual segment. We estimated the reproducibility of feature extraction using inter-class correlation coefficients (ICCs) and ICCs value greater than 0.80 indicates good reproducibility ${ }^{37}$. Additionally, 30 patients were randomly selected from each CT scanner to build the CT scanners set for calculating the intra-/interclass correlation coefficients (ICCs).

\section{Feature extraction}

Radiomic features were extracted and selected using PyRadiomics software ${ }^{38}$. Seven classes of radiomics features (first-order histogram, 3D morphologic, grey level co-existence matrix (GLCM), grey level range-matrix (GLRM), grey level size zone matrix (GLSZM), neighbouring grey tone difference matrix (NGTDM), and grey level dependence matrix (GLDM) features) were extracted from original and filtered images (wavelet and Laplacian of Gaussian). Finally, 1037 3D radiomics features were extracted from each phase of triphasic enhanced CT. The specific definitions and descriptions of the features are demonstrated in the supplementary materials.

\section{Feature selection and radiomics prediction model building}

After radiomics features extraction, all missing data in the training cohort were replaced by median value and z-score normalisation was performed on each feature, and the same preprocessing procedure was applied to the validation cohort. After features preprocessing, the most importance features were selected to predict KRAS mutation using a three-step procedure. Firstly, univariate analysis was performed for feature selection to retain the feature with $\mathrm{p}<0.05$ to enter the following process. Secondly, the Boruta method ${ }^{39}$ was used to retain the features that closely associated with KRAS mutations. Finally, multivariable stepwise regression further eliminates irrelevant features and retains the most informative features. A ten times five-fold cross-validation was applied to avoid overfitting and to identify the model with the best performance.

Three radiomics models (AP model, VP model, and DP model)were established based on the above radiomics signatures in triphasic phases enhanced CT images. For example, VP model was built based on VP features in 3D segmentation patterns (three features). Further, the 3D-combined model was built based on $\mathrm{AP}, \mathrm{VP}$, and DP fusion features in 3D segmentation patterns (eleven features).

\section{Clinical and combined model construction}

For clinical and imaging characteristics, the Chi-squared test or Fisher's exact test were used to compare the differences in sex, tumor location, CEA, CA125 and CA199, cT stage, and $\mathrm{CN}$ stage, while the Student's t-test or Mann-Whitney $\mathrm{U}$ test were used to compare the differences in age and maximum diameter between mutated KRAS and wild-type KRAS groups in the training cohorts. Generally, $P$ values $<0.05$ (two-sided) were considered statistically significant. We performed multivariable analyses to identify the most important features. A clinical model was established based on the inclusion of selected features.

A clinical-radiomics fusion model was developed based on correlated clinical risk factors, strong correlated imaging characteristics, and radiomics features to verify whether the combination of radiomics signatures and clinical factors could improve performance in the prediction of KRAS mutations. Two steps were used to build the fusion model in this study. First, AP, VP and DP models were compared to determine the enhancement phase with the best KRAS mutation prediction performance. Secondly, Random Forest (RF) algorithm was used to combine clinical factors, imaging characteristics, and the radiomics features of the best predictive performance phase to construct a clinical-radiomics fusion model in the training cohort, and the discriminant ability of the fusion model was evaluated by AUC value in the validation cohort.

\section{Statistical analysis}

All statistical analyses were conducted using the R statistical software package (version 3.6.3; http://www.Rproject.org). The Student's t-test, Mann-Whitney U test, and chi-squared test or Fisher's exact test was used to compare continuous and categorical variables, as appropriate. A two-sided $P$ value $<0.05$ was considered statistically significant. The intra-/interclass correlation coefficients (ICCs) were used to calculate the consistency of measurements between the two radiologists and different CT scanners. ROC analysis was used to evaluate the predictive accuracy of the different models. The AUC value and $95 \%$ confidence interval (Cl), accuracy, sensitivity, specificity, positive predictive value (PPV), and negative predictive value (NPV) were also calculated.

\section{Declarations}

\section{Acknowledgements}

This study received funding from the National Natural Science Foundation of China (82071872); Open Fund project of Key Laboratory of Medical Imaging of Gansu Province (GSYX202009); Science and Technology Project of Qinghai Province (2017-SF-158) and Qinghai Provincial Key Clinical Specialty

Construction Project.

\section{Author Contributions}

Conception and design: Junlin Zhou, Haihua Bao, Yuntai Cao,

Collection and assembly of the data: Yuntai Cao, Jing Zhang, Lele Huang, Zhiyong Zhao, Guojin Zhang, Yue Xing

Development of the methodology: Jialiang Ren

Data analysis and interpretation: All authors

Manuscript writing: All authors 
Final approval of the manuscript: All authors

\section{Data Accessibility}

Data are available from the corresponding author upon reasonable request.

\section{Conflicts of interest}

The authors declare no conflicts of interest.

\section{References}

1. Bray F, Ferlay J, Soerjomataram I, Siegel RL, Torre LA, Jemal A. Global cancer statistics 2018: GLOBOCAN estimates of incidence and mortality worldwide for 36 cancers in 185 countries. CA Cancer J Clin 2018;68:394-424.

2. Dekker E, Tanis PJ, Vleugels JLA, Kasi PM, Wallace MB. Colorectal cancer. Lancet 2019;394:1467-80.

3. De Roock W, Claes B, Bernasconi D, De Schutter J, Biesmans B, Fountzilas G, Kalogeras KT, Kotoula V, Papamichael D, Laurent-Puig P, Penault-Llorca F, Rougier P, Vincenzi B, Santini D, Tonini G, Cappuzzo F, Frattini M, Molinari F, Saletti P, De Dosso S, Martini M, Bardelli A, Siena S, Sartore-Bianchi A, Tabernero J, Macarulla T, Di Fiore F, Gangloff AO, Ciardiello F, Pfeiffer P, Qvortrup C, Hansen TP, Van Cutsem E, Piessevaux H, Lambrechts D, Delorenzi M, Tejpar S. Effects of KRAS, BRAF, NRAS, and PIK3CA mutations on the efficacy of cetuximab plus chemotherapy in chemotherapy-refractory metastatic colorectal cancer: a retrospective consortium analysis. Lancet Oncol 2010;11:753-62.

4. Van Cutsem E, Lenz HJ, Köhne CH, Heinemann V, Tejpar S, Melezínek I, Beier F, Stroh C, Rougier P, van Krieken JH, Ciardiello F. Fluorouracil, leucovorin, and irinotecan plus cetuximab treatment and RAS mutations in colorectal cancer. J Clin Oncol 2015;33:692-700.

5. Watanabe T, Kobunai T, Yamamoto Y, Matsuda K, Ishihara S, Nozawa K, linuma H, Shibuya H, Eshima K. Heterogeneity of KRAS status may explain the subset of discordant KRAS status between primary and metastatic colorectal cancer. Dis Colon Rectum 2011;54:1170-8.

6. Sundström M, Edlund K, Lindell M, Glimelius B, Birgisson H, Micke P, Botling J. KRAS analysis in colorectal carcinoma: analytical aspects of Pyrosequencing and allele-specific PCR in clinical practice. BMC Cancer 2010;10:660.

7. Wang S, Shi J, Ye Z, Dong D, Yu D, Zhou M, Liu Y, Gevaert O, Wang K, Zhu Y, Zhou H, Liu Z, Tian J. Predicting EGFR mutation status in lung adenocarcinoma on computed tomography image using deep learning. Eur Respir J 2019;53.

8. Lovinfosse P, Polus M, Van Daele D, Martinive P, Daenen F, Hatt M, Visvikis D, Koopmansch B, Lambert F, Coimbra C, Seidel L, Albert A, Delvenne P, Hustinx R. FDG PET/CT radiomics for predicting the outcome of locally advanced rectal cancer. Eur J Nucl Med Mol Imaging 2018;45:365-75.

9. Shin YR, Kim KA, Im S, Hwang SS, Kim K. Prediction of KRAS Mutation in Rectal Cancer Using MRI. Anticancer Res 2016;36:4799-4804.

10. He K, Liu X, Li M, Li X, Yang H, Zhang H. Noninvasive KRAS mutation estimation in colorectal cancer using a deep learning method based on CT imaging. BMC Med Imaging 2020;20:59.

11. Yang L, Dong D, Fang M, Zhu Y, Zang Y, Liu Z, Zhang H, Ying J, Zhao X, Tian J. Can CT-based radiomics signature predict KRAS/NRAS/BRAF mutations in colorectal cancer? Eur Radiol 2018;28:2058-67.

12. Chu E. An update on the current and emerging targeted agents in metastatic colorectal cancer. Clin Colorectal Cancer 2012;11.

13. Berthélemy P, Bouisson M, Escourrou J, Vaysse N, Rumeau JL, Pradayrol L. Identification of K-ras mutations in pancreatic juice in the early diagnosis of pancreatic cancer. Ann Intern Med 1995;123:188-91.

14. Van Laethem JL, Vertongen P, Deviere J, Van Rampelbergh J, Rickaert F, Cremer M, Robberecht P. Detection of c-Ki-ras gene codon 12 mutations from pancreatic duct brushings in the diagnosis of pancreatic tumours. Gut 1995;36:781-7.

15. Karapetis CS, Khambata-Ford S, Jonker DJ, O'Callaghan CJ, Tu D, Tebbutt NC, Simes RJ, Chalchal H, Shapiro JD, Robitaille S, Price TJ, Shepherd L, Au HJ, Langer C, Moore MJ, Zalcberg JR. K-ras mutations and benefit from cetuximab in advanced colorectal cancer. N Engl J Med 2008;359:1757-65.

16. Chen SW, Chiang HC, Chen WT, Hsieh TC, Yen KY, Chiang SF, Kao CH. Correlation between PET/CT parameters and KRAS expression in colorectal cancer. Clin Nucl Med 2014;39:685-9.

17. Cho A, Jo K, Hwang SH, Lee N, Jung M, Yun M, et al. Correlation between KRAS mutation and F-FDG uptake in stage IV colorectal cancer. Abdom Radiol (NY) 2017;42:1621-6.

18. Krikelis D, Skoura E, Kotoula V, Rondogianni P, Pianou N, Samartzis A, Xanthakis I, Fountzilas G, Datseris IE. Lack of association between KRAS mutations and 18F-FDG PET/CT in Caucasian metastatic colorectal cancer patients. Anticancer Res 2014;34:2571-9.

19. Cui Y, Liu H, Ren J, Du X, Wang D. Development and Validation of a MRI Based Radiomics Signature for Prediction of KRAS Mutation in Rectal Cancer. Eur Radiol 2020; 30(4):1948-58.

20. Liu H, Zhang C, Wang L, Luo R, Li J, Zheng H, Yin Q, Zhang Z, Duan S, Li X, Wang D. MRI radiomics analysis for predicting preoperative synchronous distant metastasis in patients with rectal cancer. Eur Radiol 2019;29:4418-26.

21. Lubner MG, Smith AD, Sandrasegaran K, et al. CT Texture Analysis: Definitions, Applications, Biologic Correlates, and Challenges. Radiographics. 2017;37(5):1483-1503.

22. Ganeshan B, Panayiotou E, Burnand K, Dizdarevic S, Miles K. Tumour heterogeneity in non-small cell lung carcinoma assessed by CT texture analysis: a potential marker of survival. Eur Radiol. 2012;22(4):796-802.

23. Bashir U, Siddique MM, Mclean E, Goh V, Cook GJ. Imaging Heterogeneity in Lung Cancer: Techniques, Applications, and Challenges. AJR Am J Roentgenol. 2016;207(3):534-543. 
24. Oh JE, Kim MJ, Lee J, Hur BY, Kim B, Kim DY, Baek JY, Chang HJ, Park SC, Oh JH, Cho SA, Sohn DK. Magnetic Resonance-Based Texture Analysis Differentiating KRAS Mutation Status in Rectal Cancer. Cancer Res Treat 2020;52:51-9.

25. Cao Y, Zhang G, Zhang J, et al. Predicting Microsatellite Instability Status in Colorectal Cancer Based on Triphasic Enhanced Computed Tomography Radiomics Signatures: A Multicenter Study. Front Oncol. 2021;11:687771.

26. Fu X, Huang Y, Fan X, et al. Demographic trends and KRAS/BRAF mutations in colorectal cancer patients of South China: A single-site report. Int J Cancer. 2019;144:2109-2117.

27. Selcukbiricik F, Bilici A, Tural D, Erdamar S, Soyluk O, Buyukunal E, Demirelli F, Serdengecti S. Are high initial CEA and CA 19-9 levels associated with the presence of K-ras mutation in patients with metastatic colorectal cancer? Tumour Biol 2013;34:2233-9.

28. Li W, Qiu T, Ling Y, Guo L, Li L, Ying J. Molecular pathological epidemiology of colorectal cancer in Chinese patients with KRAS and BRAF mutations. Oncotarget 2015;6:39607-13.

29. Li W, Qiu T, Zhi W, Shi S, Zou S, Ling Y, Shan L, Ying J, Lu N. Colorectal carcinomas with KRAS codon 12 mutation are associated with more advanced tumor stages. BMC Cancer 2015;15:340.

30. Bazan V, Agnese V, Corsale S, Calò V, Valerio MR, Latteri MA, Vieni S, Grassi N, Cicero G, Dardanoni G, Tomasino RM, Colucci G, Gebbia N, Russo A; Gruppo Oncologico dell'Italia Meridionale (GOIM). Specific TP53 and/or Ki-ras mutations as independent predictors of clinical outcome in sporadic colorectal adenocarcinomas: results of a 5-year Gruppo Oncologico dell'Italia Meridionale (GOIM) prospective study. Ann Oncol 2005;16 Suppl 4:iv50-5.

31. Li M, Zhang J, Dan Y, Yao Y, Dai W, Cai G, Yang G, Tong T. A clinical-radiomics nomogram for the preoperative prediction of lymph node metastasis in colorectal cancer. J Transl Med 2020;18:46.

32. Jeon C-H, Lee H-I, Shin I-H, Park J-W. Genetic alterations of APC, K-ras, p53, MSI, and MAGE in Korean colorectal cancer patients. International journal of colorectal disease. Int J Colorectal Dis 2008;23:29-35.

33. Lee JH, Jeong YK, Kim DH, Go BK, Woo YJ, Ham SY, Yang SO. Two-phase helical CT for detection of early gastric carcinoma: importance of the mucosal phase for analysis of the abnormal mucosal layer. J Comput Assist Tomogr 2000;24:777-82.

34. Cao Y, Zhang G, Bao H, et al. Development of a dual-energy spectral CT based nomogram for the preoperative discrimination of mutated and wild-type KRAS in patients with colorectal cancer. Clin Imaging. 2021;69:205-212.

35. Jia Y, Zhai B, He T, Yu Y, Yu N, Duan H, Yang C, Zhang X. The Application of a New Model-Based Iterative Reconstruction in Low-Dose Upper Abdominal CT. Acad Radiol 2019;26:e275-83.

36. Amin MB, Greene FL, Edge SB, Compton CC, Gershenwald JE, Brookland RK, Meyer L, Gress DM, Byrd DR, Winchester DP. The Eighth Edition AJCC Cancer Staging Manual: Continuing to build a bridge from a population-based to a more "personalized" approach to cancer staging. CA Cancer J Clin 2017;67:939.

37. Aerts HJ, Velazquez ER, Leijenaar RT, Parmar C, Grossmann P, Carvalho S, Bussink J, Monshouwer R, Haibe-Kains B, Rietveld D, Hoebers F, Rietbergen MM, Leemans CR, Dekker A, Quackenbush J, Gillies RJ, Lambin P. Decoding tumour phenotype by noninvasive imaging using a quantitative radiomics approach. Nat Commun 2014;5:4006.

38. Van Griethuysen JJM, Fedorov A, Parmar C, Hosny A, Aucoin N, Narayan V, Beets-Tan RGH, Fillion-Robin JC, Pieper S, Aerts HJWL. Computational radiomics system to decode the radiographic phenotype. Cancer Res 2017;77:e104-7.

39. Degenhardt F, Seifert S, Szymczak S. Evaluation of variable selection methods for random forests and omics data sets. Brief Bioinform 2019;20:492-503.

\section{Figures}




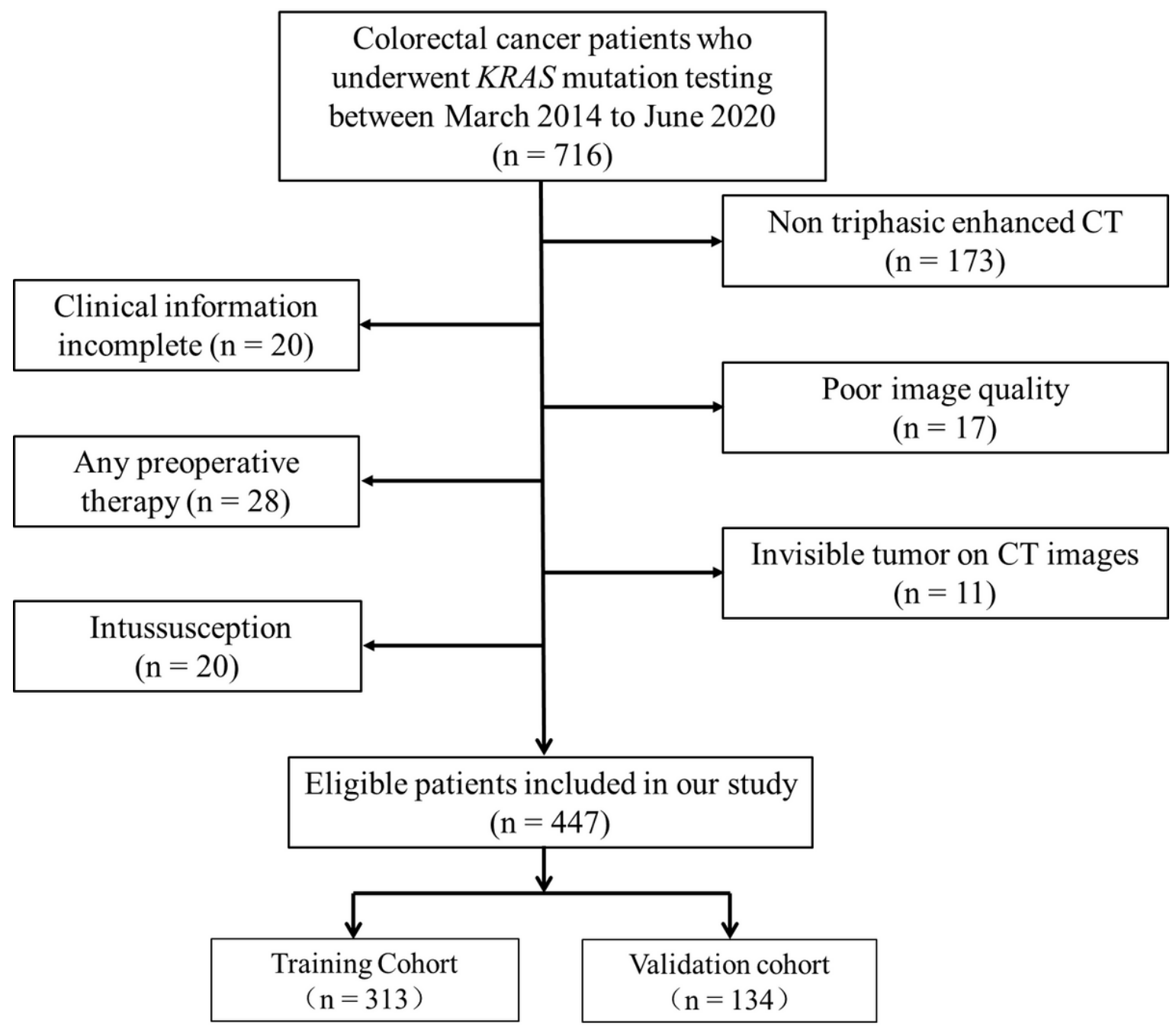

Figure 1

Flow diagram of the recruitment pathway. 

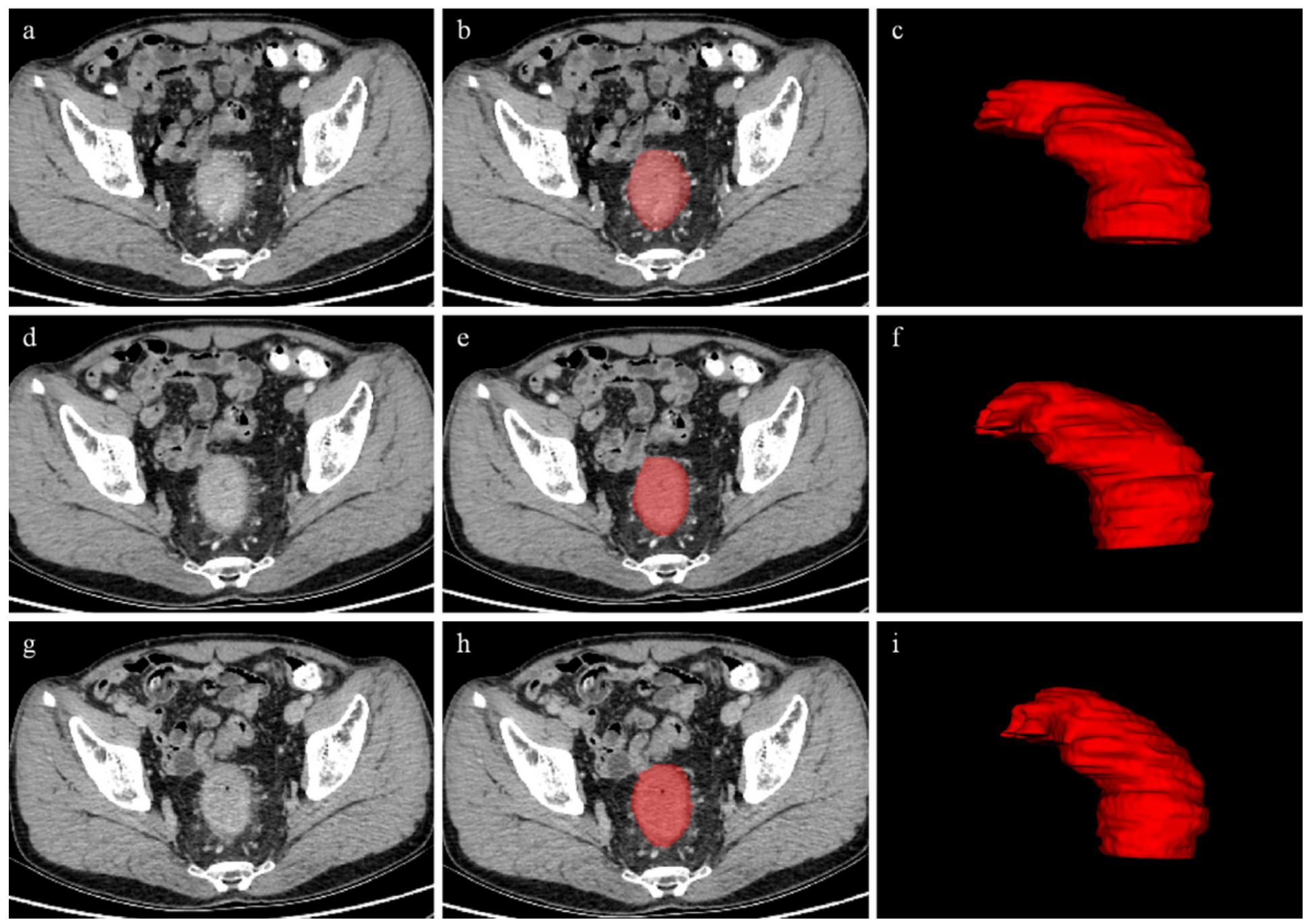

Figure 2

A 58-year-old female with rectal cancer was pathologically confirmed to have KRAS mutation. The example of tumor delineation and segmentation are shown on the arterial (a-c), venous ( $d-f)$, and delayed phase $(g-i)$. Fig a, $d$ and g represents the delineation of rectal cancer in the arterial, venous, and delayed phase, respectively. Fig b, e and $\mathrm{h}$ represents the corresponding manual region of interest (ROI) in the arterial, venous, and delayed phase, respectively. Fig c, $f$ and $i$ represents the whole volume extracted from the same rectal tumor. 

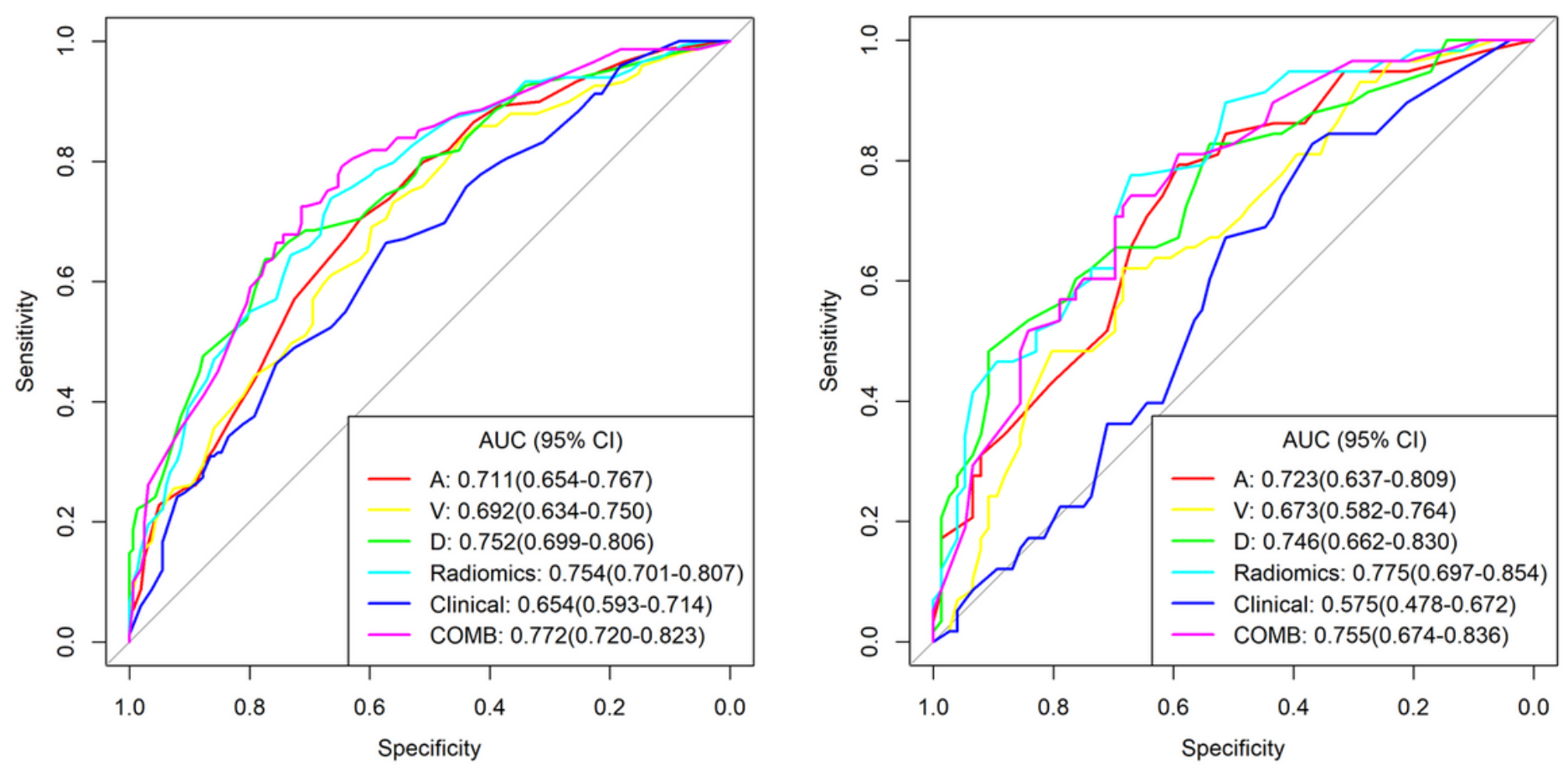

\section{Figure 3}

ROC curves of the different models in training (A) and validation cohorts (B). AUC, area under the curve; A, radiomics model of arterial phase; D, radiomics model of delayed phase; $\mathrm{V}$, radiomics model of venous phase; Radiomics, radiomics model of fusion of arterial phase, delayed phase and venous phase features; $C O M B$, fusion of clinical risk factors and radiomics features of delayed phase.
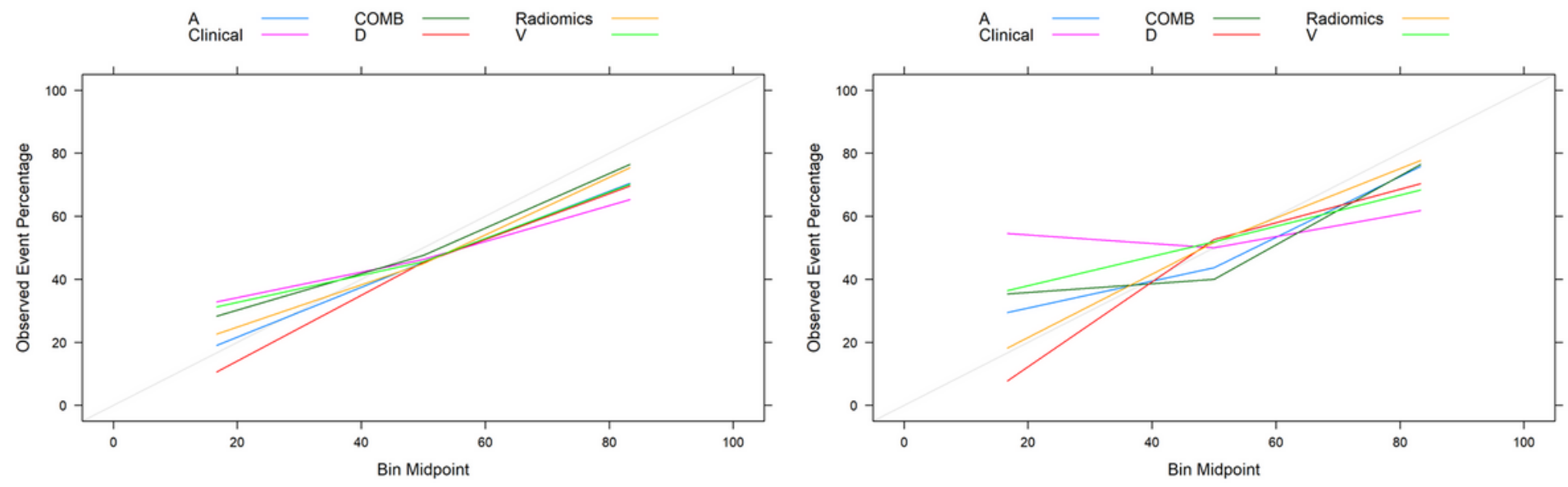

\section{Figure 4}

Calibration curves of the different models in training $(A)$ and validation cohorts $(B)$. A, radiomics model of arterial phase; $D$, radiomics model of delayed phase; $\mathrm{V}$, radiomics model of venous phase; Radiomics, radiomics model of fusion of arterial phase, delayed phase and venous phase features; COMB, fusion of clinical risk factors and radiomics features of delayed phase. 

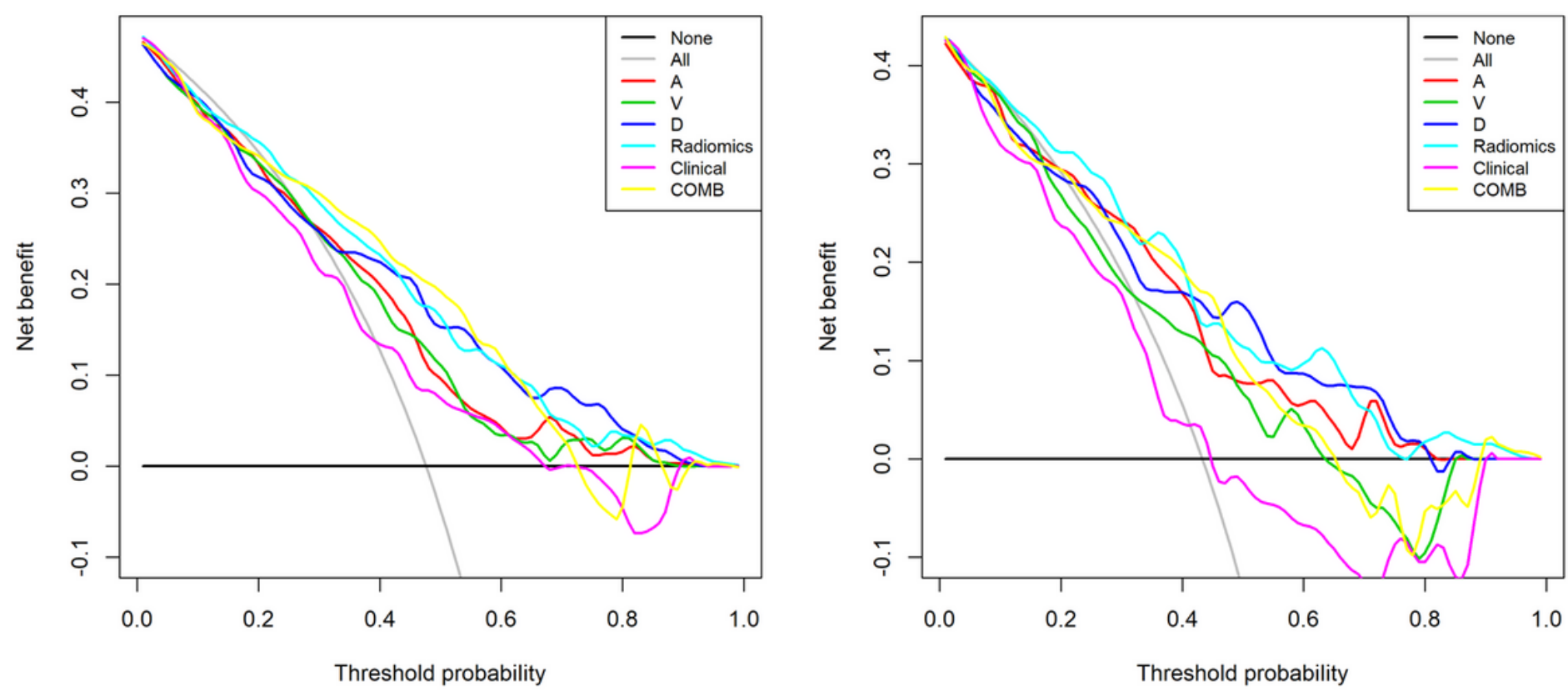

Figure 5

Decision curve analysis of different models in training (A) and validation cohorts (B). A, radiomics model of arterial phase; $D$, radiomics model of delayed phase; $\mathrm{V}$, radiomics model of venous phase; Radiomics, radiomics model of fusion of arterial phase, delayed phase and venous phase features; $C O M B$, fusion of clinical risk factors and radiomics features of delayed phase.

\section{Supplementary Files}

This is a list of supplementary files associated with this preprint. Click to download.

- SupplementaryMaterials.docx 